\title{
Formation of creases on the surfaces of elastomers and gels
}

\section{Citation}

Hong, Wei, Xuanhe Zhao, and Zhigang Suo. 2009. "Formation of Creases on the Surfaces of Elastomers and Gels." Applied Physics Letters 95 (11): 111901. https:// doi.org/10.1063/1.3211917.

\section{Permanent link}

http://nrs.harvard.edu/urn-3:HUL.InstRepos:41461294

\section{Terms of Use}

This article was downloaded from Harvard University's DASH repository, and is made available under the terms and conditions applicable to Other Posted Material, as set forth at http:// nrs.harvard.edu/urn-3:HUL.InstRepos:dash.current.terms-of-use\#LAA

\section{Share Your Story}

The Harvard community has made this article openly available.

Please share how this access benefits you. Submit a story.

Accessibility 


\title{
Formation of creases on the surfaces of elastomers and gels
}

\author{
Wei Hong, ${ }^{1}$ Xuanhe Zhao, ${ }^{2}$ and Zhigang Suo ${ }^{2, a)}$ \\ ${ }^{1}$ Department of Aerospace Engineering, Iowa State University, Ames, Iowa 50011, USA \\ ${ }^{2}$ School of Engineering and Applied Sciences, Harvard University, Cambridge, Massachusetts 02138, USA
}

(Received 9 July 2009; accepted 31 July 2009; published online 14 September 2009)

\begin{abstract}
When a block of an elastomer is bent, the compressed surface may form a crease. The critical strain for creasing measured experimentally is known to disagree with that predicted by linear perturbation analysis. This paper calculates the critical strain by comparing the elastic energy in a creased body and that in a smooth body. This difference in energy is expressed by a scaling relation. Critical conditions for creasing are determined for elastomers subject to general loads and gels swelling under constraint. The theoretical results are compared with existing experimental observations. (C) 2009 American Institute of Physics. [doi:10.1063/1.3211917]
\end{abstract}

When a block of an elastomer or a gel is bent, the compressed surface remains smooth initially, but then suddenly forms a crease. ${ }^{1,2}$ Similar creases have also been observed when a gel imbibes a solvent and swells. ${ }^{3-6}$ The creases are often undesirable in applications. For example, repetitive folding and unfolding of creases on the side walls of a tire may result in fatigue failure. In biomedical applications, inorganic materials are often coated with thin layers of gels, where creases may damage the coatings.

While creases are commonplace in daily life (e.g., Fig. 1), their scientific understanding has been intriguing. Biot's linear perturbation analysis showed that the homogenous deformation in a rubber becomes unstable when compressed to a critical strain, $\varepsilon_{\text {biot }}=0.46 .^{7}$ This theoretical value, however, exceeds the experimental value, $\varepsilon_{\exp }=0.35 .^{1,2}$ This discrepancy was not addressed in the theoretical literature ${ }^{8-10}$ until very recently, when crease was identified as a distinct mode of instability, different from that analyzed by Biot. ${ }^{11,12} \mathrm{By}$ following a limiting process, Refs. 11 and 12 show that the two modes of instability occur at different critical strains.

The difference between the two modes of instability may be understood as follows. Subject to a load, a rubber develops a field of deformation. Of all possible fields of deformation, the stable field minimizes the free energy of the rubber. Instead of searching among all possible fields, Biot carried out a linear perturbation analysis, which in effect restricted his search among fields that deviate from the homogenous state by infinitesimal strains. For a crease, however, a stripe of the surface folds and makes contact to itself. While the region affected by the initial crease is small, the amplitude deviates from the homogeneous state by large strains.

Here we analyze the creasing instability by comparing the elastic energy in a creased elastomer and that in a smooth elastomer. We express this difference in energy by a scaling relation. Critical conditions for creasing are obtained for both elastomers under general loads and gels swelling under constraint. The theoretical results are compared to available experimental data.

Figure 2 sketches the model to be analyzed. An undeformed block of elastomer is taken to be the reference state [Fig. 2(a)]. Attached on the surface are three markers: $A^{\prime}, O$, and A, separated by an arbitrary length $L$. The block is then

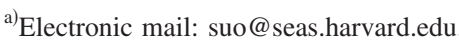

compressed by a strain $\varepsilon$, defined as the shortening divided by the original length of the block. The elastomer may either deform to a homogenous state [Fig. 2(b)], or form a crease [Fig. 2(c)]. In the creased state, the surface folds at $\mathrm{O}$, and stripe $\mathrm{OA}^{\prime}$ contacts with stripe OA.

We analyze the inhomogeneous deformation of the creased state in Fig. 2(c) by using the finite-element software, ABAQUS 6.7.1 (standard). The elastomer is taken to deform under the plane-strain conditions, and obey the neoHookean model with the free-energy density ${ }^{13}$

$$
W=\frac{\mu}{2}\left(\lambda_{1}^{2}+\lambda_{2}^{3}+\lambda_{3}^{2}-3\right)
$$

where $\mu$ is the shear modulus, and $\lambda_{1}, \lambda_{2}$, and $\lambda_{3}$ are the principal stretches. The elastomer is taken to be incompressible, so that $\lambda_{1} \lambda_{2} \lambda_{3}=1$. Due to symmetry, only the right half of the block is modeled. The block is compressed by a uniform displacement on a boundary far away from the crease. A displacement is specified on the segment OA of the top surface, so that it rotates to the vertical position illustrated in Fig. 2(c). The vertical displacement of OA is not prescribed and is solved as a part of the boundary-value problem. We find that a block of size $50 \times 50 \mathrm{~L}$ is large enough to mimic a semi-infinite block.

The size of the initial crease is taken to be much smaller than the size of the block, so that $L$ is the only length in the

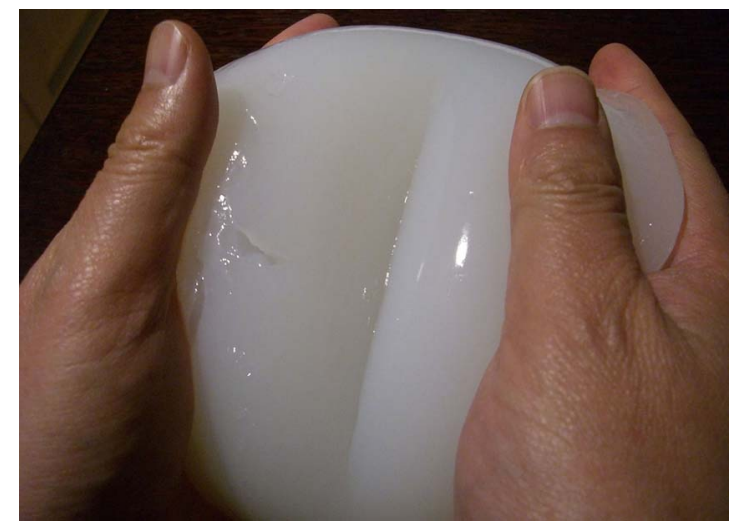

FIG. 1. (Color online) A crease formed on the compressed surface of a popular Chinese food, Liang Fen (a starch gel). 


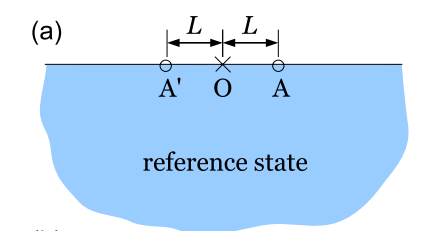

(b)

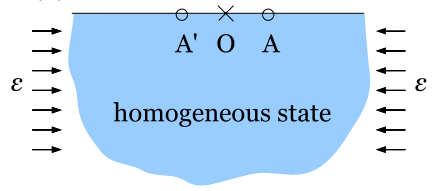

(c)

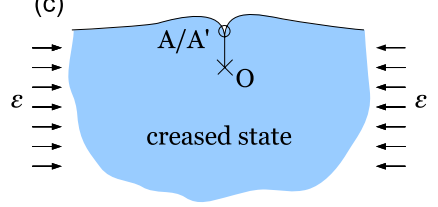

FIG. 2. (Color online) Schematic a semi-infinite block of elastomer. (a) The undeformed elastomer is taken to be the reference state. (b) The block is in a state of homogeneous deformation. (c) The block is prescribed with a crease.

boundary-value problem. Let $U$ be the elastic energy per unit thickness of the creased body minus that in the smooth body. Dimensional considerations dictate that

$$
U=\mu L^{2} f(\varepsilon),
$$

where $f(\varepsilon)$ is a dimensionless function of the applied strain. Figure 3 plots the results calculated by using the finite element method. In the absence of the applied strain, the homogenous state has no elastic energy, but the creased state has elastic energy, so that $f(0)>0$. When a small compressive strain is applied, the crease helps to relieve some of the compression, so that $f(\varepsilon)$ is a decreasing function. At a critical strain, $\varepsilon_{c}$, the creased state has the same energy as the homogenous state, $f\left(\varepsilon_{c}\right)=0$. Our numerical calculation gives the value of the critical strain $\varepsilon_{c} \approx 0.35$.

The curve on Fig. 3 stops at the critical point. No state of equilibrium exists when $\varepsilon>\varepsilon_{c}$. A semi-infinite block does not have any length scale. Following Eq. (2), when $\varepsilon>\varepsilon_{c}$, the creased state has a lower energy than the homogenous state for any depth $L$ of the crease, so that the crease will extend indefinitely without reaching equilibrium. In a finite block constrained at the bottom, such as a coating on a rigid

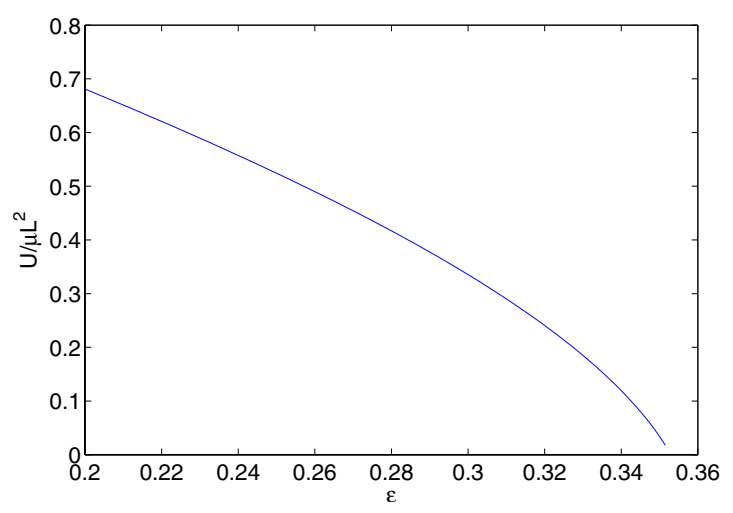

FIG. 3. (Color online) The difference in elastic energy between the creased state and the homogeneous state, calculated with ABAQUS. The critical value of applied strain is approximately $\varepsilon_{c} \approx 0.35$.

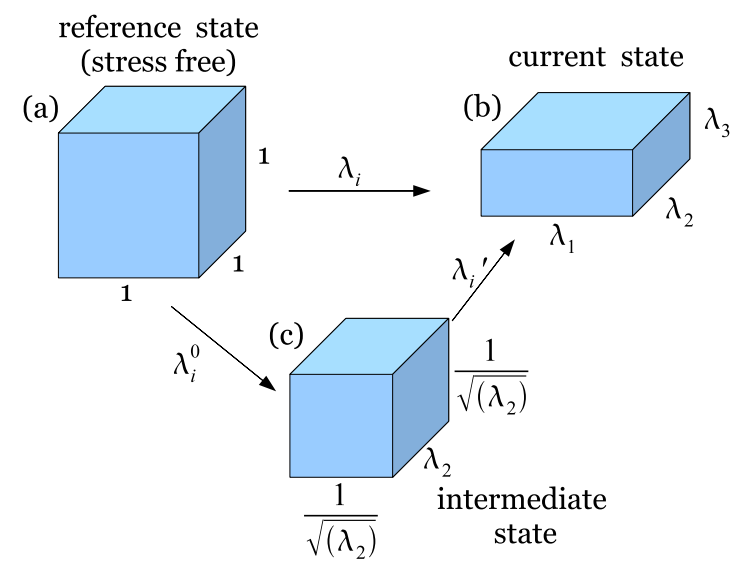

FIG. 4. (Color online) A generalized plane-strain problem and its equivalent plane-strain problem.

substrate, the crease does equilibrate at a finite depth. The equilibrium depth of a crease in a finite body is not studied in this paper.

The predicted critical strain, $\varepsilon_{c}=0.35$, agrees with the experimental value. ${ }^{1,2}$ The agreement is remarkable, considering that the theory is based on an idealized material model (the neo-Hookean model), and that the two sets of experiments were carried out using different materials. The agreement, however, is not fortuitous. In a limiting case, when a semi-infinite block of an incompressible material is folded along a straight line on the surface, such that one half of the surface contacts the other half of the surface, the state of the deformation is fully determined by geometric considerations, giving stretch $1 / \sqrt{2}$ in the radial direction, and stretch $\sqrt{2}$ in the circumferential direction. ${ }^{14}$ The deformation is finite but modest, and should be well represented by the neo-Hookean model.

We next consider an elastomer under general loads. Consider a semi-infinite block of an elastomer in a homogeneous state of deformation, with $\lambda_{1}$ and $\lambda_{2}$ being the stretches in the directions parallel to the surface of the block, and $\lambda_{3}$ being the stretch in the direction normal to the surface. The compression in direction 1 is taken to be more severe than that in direction 2 , so that when a crease forms in a plane normal to direction 1 , leaving $\lambda_{2}$ unchanged. That is, the crease is in a state of generalized plane strain.

This generalized plane-strain problem $\left(\lambda_{2}=\right.$ constant $)$ may be transformed into an equivalent plane-strain problem $\left(\lambda_{2}=1\right)$. Figure 4 illustrates that a unit cube in the reference state (a) deforms by principal stretches $\lambda_{i}$ to the current state, (b). Imagine an intermediate state, and (c) of stretches $\lambda_{2}^{0}$ $=\lambda_{2}$ and $\lambda_{1}^{0}=\lambda_{3}^{0}=1 / \sqrt{\lambda_{2}}$. Now regard the intermediate state as another reference state, and let $\lambda_{i}^{\prime}$ be the stretches in the current state measured with respect to the intermediate state, so that

$$
\lambda_{1}=\lambda_{1}^{\prime} / \sqrt{\lambda_{2}}, \quad \lambda_{3}=\lambda_{3}^{\prime} / \sqrt{\lambda_{2}}, \quad \lambda_{2}=\lambda_{2} .
$$

Substituting Eq. (3) into Eq. (1), we obtain that

$$
W=\frac{\mu}{2 \lambda_{2}}\left[\left(\lambda_{1}^{\prime}\right)^{2}+\left(\lambda_{3}^{\prime}\right)^{2}\right]+\frac{\mu}{2}\left(\lambda_{2}^{2}-3\right) .
$$

Because $\lambda_{2}$ is a prescribed constant, the last term in Eq. (4) does not contribute to the difference in the energy between the creased body and the smooth body. The first two terms in 
Eq. (4), together with equations of equilibrium and kinematics, governs the deformation from the intermediate state to the current state, which is a plane-strain deformation. Consequently, the generalized plane-strain problem is equivalent to a plane strain problem with an effective shear modulus $\mu / \lambda_{2}$.

The critical strain $\varepsilon_{c}=0.35$ was obtained under the plane strain conditions. This critical condition corresponds to $\lambda_{1}$ $=1-\varepsilon_{c}=0.65$, and is applicable to the deformation from (c) to (b) in Fig. 4, giving $\lambda_{1}^{\prime}=0.65$. According to Eq. (3), this condition corresponding to $\lambda_{1} \sqrt{\lambda_{2}}=0.65$. Recall that $\lambda_{1} \lambda_{2} \lambda_{3}$ $=1$, we write the critical condition as

$$
\lambda_{3} / \lambda_{1}=2.4 \text {. }
$$

The same procedure transforms Biot's critical strain $\varepsilon_{\text {biot }}$ $=0.46$ to an expression valid under the generalized planestrain conditions,

$$
\lambda_{3} / \lambda_{1}=3.4 \text {. }
$$

Equations (5) and (6) are valid under the generalized planestrain conditions for any arbitrary value of $\lambda_{2}$.

Creases are often observed on the surface of a swelling gel. ${ }^{3-6}$ Consider a gel bonded on a rigid substrate, and swells in the direction normal to the surface. Let $\eta$ be the ratio of the thickness of the swollen gel and that of the initial gel. Imagine that we remove the constraint, and the gel instantaneously deforms into a stress-free state, with an isotropic stretch $\eta^{1 / 3}$. From this imaginary state to the constrained swollen state, the gel stretches by $\lambda_{1}=\lambda_{2}=\eta^{-1 / 3}$ and $\lambda_{3}$ $=\eta^{2 / 3}$. Inserting these stretches into Eqs. (5) and (6), we obtain that the critical condition is $\eta_{c}=2.4$ for creasing, and is $\eta_{\text {biot }}=3.4$ for linear perturbation. Several sets of experimental data are found in the literature, ${ }^{4-6}$ giving $\eta_{\exp }=2.0-3.7$.

The above calculation assumes that creasing is a fast process and solvent in the gel has no time to migrate, so that creasing of the gel resembles that of an incompressible elastomer. ${ }^{15}$ Further study is needed to understand the variations in the experimental data, and to examine the consequence of solvent migration.

In summary, by comparing the energy of the creased state and that of the homogeneous state, the theory predicts that the crease lower the elastic energy of the body when compressed by $35 \%$. This critical strain is below that predicted by Biot's linear perturbation analysis, and agrees with experimental observations. Critical conditions for creasing are also obtained for an elastomer under general loading, and for a swelling gel attached to a substrate.

This work was supported by the NSF through a grant on Soft Active Materials (Grant No. CMMI-0800161) and through the MRSEC at Harvard University. The manuscript has been revised to incorporate comments made by the reviewer of the paper, as well as those made by Rui Huang on an early draft posted at http://imechanica.org/node/5999.

${ }^{1}$ A. N. Gent and I. S. Cho, Rubber Chem. Technol. 72, 253 (1999).

${ }^{2}$ A. Ghatak and A. L. Das, Phys. Rev. Lett. 99, 076101 (2007).

${ }^{3}$ T. Tanaka, S.-T. Sun, Y. Hirokawa, S. Katayama, J. Kucera, Y. Hirose, and T. Amiya, Nature (London) 325, 796 (1987).

${ }^{4}$ E. Southern and A. G. Thomas, J. Polym. Sci. A 3, 641 (1965).

${ }^{5}$ H. Tanaka, H. Tomita, A. Takasu, T. Hayashi, and T. Nishi, Phys. Rev. Lett. 68, 2794 (1992)

${ }^{6}$ V. Trujillo, J. Kim, and R. C. Hayward, Soft Matter 4, 564 (2008).

${ }^{7}$ M. A. Biot, Appl. Sci. Res., Sect. A 12, 168 (1963).

${ }^{8}$ T. Hwa and M. Kardar, Phys. Rev. Lett. 61, 106 (1988).

${ }^{9}$ H. Tanaka and T. Sigehuzi, Phys. Rev. E 49, R39 (1994).

${ }^{10}$ A. Onuki, Phys. Rev. A 39, 5932 (1989).

${ }^{11}$ E. B. Hohlfeld, Ph.D. thesis, Harvard University, 2008.

${ }^{12}$ E.B. Hohlfeld and L. Mahadevan (unpublished).

${ }^{13}$ L. R. G. Treloar, The Physics of Rubber Elasticity (Oxford University Press, New York, 1975).

${ }^{14}$ M. Singh and A. C. Pipkin, Z. Angew. Math. Phys. 16, 706 (1965).

${ }^{15}$ W. Hong, X. Zhao, J. Zhou, and Z. Suo, J. Mech. Phys. Solids 56, 1779 (2008). 